\title{
APPROXIMATION OF RANDOM VARIABLES BY FUNCTIONALS OF THE INCREMENTS OF A FRACTIONAL BROWNIAN MOTION
}

UDC 519.21

\author{
G. M. SHEVCHENKO AND T. O. SHALAIKO
}

\begin{abstract}
A lower estimate is given for the accuracy of approximation of random variables by functionals of the increments of a fractional Brownian motion with Hurst index $H>\frac{1}{2}$.
\end{abstract}

\section{INTRODUCTION}

A centered Gaussian stochastic process with the covariance function

$$
R_{H}(t, s)=\frac{1}{2}\left(t^{2 H}+s^{2 H}-|t-s|^{2 H}\right)
$$

is called a fractional Brownian motion with Hurst index $H>\frac{1}{2}$ and is denoted by

$$
B^{H}=\left\{B_{t}^{H}, t \geq 0\right\} .
$$

The fractional Brownian motion $B^{H}$ with $H>\frac{1}{2}$ possesses the properterty of strong dependence. Because of this property, the fractional Brownian motion is a popular model for the long range dependence phenomena in financial mathematics.

When numerically solving the stochastic differential equations driven by the fractional Brownian motion with the help of the time discretization schemes (Euler or Milstein schemes, etc.), a natural question arises on the accuracy of approximation of functionals of a solution of the stochastic differential equation (that is, the functionals of the fractional Brownian motion) by appropriate functions of the increments of the fractional Brownian motion.

It is known in the theory of the approximation of solutions of stochastic differential equations driven by the fractional Brownian motion that the precise rate of approximation of a solution of the equation in the Euler scheme is $\delta^{2 H-1}$, where $\delta$ denotes the diameter of a partition (see, for example, [3, 6, 7]). It is also proved in [3] that the precise rate of approximation in the Milstein scheme is $|\log \delta|\left(\delta^{H}+\delta^{2 H-1 / 2}\right)$. Since the approximations for both schemes are constructed from the increments of a fractional Brownian motion in the one-dimensional case, the results mentioned above provide upper bounds for the accuracy of the approximation of solutions of stochastic differential equations by functionals of the increments of a fractional Brownian motion.

The aim of this paper is to prove lower bounds for the accuracy of approximation of an arbitrary random variable by the functionals of the increments of fractional Brownian motion. It is clear that an assumption about the sufficient nondegeneracy should be imposed on the functional in this case. A similar problem is considered by Neuenkirch [8]

2010 Mathematics Subject Classification. Primary 60G22; Secondary 60G15, 65C30.

Key words and phrases. Fractional Brownian motion, Itô-Wiener expansion, an accuracy of approximation. 
for the functional being equal to the value of a solution $\left\{X_{t}, t \in[0,1]\right\}$ of the stochastic differential equation

$$
d X_{t}=a\left(X_{t}\right)+b\left(X_{t}\right) d B_{t}^{H}
$$

at the point $t=1$ under some restrictions on the coefficients of the stochastic differential equation.

Theorem 1.1. Let a centered, square integrable, and $\sigma\left\{B_{t}^{H}, t \leq T\right\}$-measurable random variable $\xi$ be such that the derivative of $f_{1}$ in the Itô-Wiener decomposition is bounded and nonvanishing in a set of positive Lebesgue measure. If the diameter $\delta=T / n$ of an equidistant partition $\pi=\{i T / n\}_{i=0}^{n}$ of the interval is sufficiently small, then

$$
\mathrm{E}\left[(\xi-F)^{2}\right] \geq C \delta^{4 H}
$$

for an arbitrary functional $F$ of the increments of a fractional Brownian motion, where $C=C(\xi)$ is a positive constant that depends on $\xi$.

\section{Preliminaries}

We briefly discuss some basic notions and results concerning the white noise space for the fractional Brownian motion; see 1 for details.

Let $\mathcal{S}(\mathbb{R})$ be the Schwartz space of rapidly decreasing functions and let $\mathcal{S}^{\prime}(\mathbb{R})$ be the dual space. On the white noise space $\left(\mathcal{S}^{\prime}(\mathbb{R}), \mathcal{B}_{\mathcal{S}^{\prime}(\mathbb{R})}\right)$, consider the measure $\mathrm{P}^{H}$, $H \in(1 / 2,1)$, defined by

$$
\int_{\mathcal{S}^{\prime}(\mathbb{R})} e^{i\langle\omega, f\rangle} d \mathrm{P}^{H}(\omega)=e^{-\|f\|_{H}^{2} / 2},
$$

where

$$
\|f\|_{H}^{2}=H(2 H-1) \iint_{\mathbb{R}^{2}} f(t) f(s)|t-s|^{2 H-2} d t d s .
$$

Such a measure exists in view of the Minlos-Sazonov theorem. On this space, we define a fractional Brownian motion $B^{H}=\left\{B_{t}^{H}, t \in[0, T]\right\}$ by letting $B_{t}^{H}=\left\langle\omega, \mathbb{I}_{[0, t]}(\cdot)\right\rangle$. Here and in what follows we assume that $H>\frac{1}{2}$. One can prove that $B^{H}$ is a centered Gaussian stochastic process with the covariance function

$$
R_{H}(t, s)=\frac{1}{2}\left(t^{2 H}+s^{2 H}-|t-s|^{2 H}\right) .
$$

By $\mathbb{F}=\left\{\mathcal{F}_{t}, t \in[0, T]\right\}$, we denote the filtration generated by the fractional Brownian motion $B^{H}$, that is, $\mathcal{F}_{t}=\sigma\left\{B_{s}^{H}, s \leq t\right\}$. For brevity, we let $\mathcal{F}_{T}=\mathcal{F}$.

The completion of the space $\mathcal{S}(\mathbb{R})$ with respect to the norm $\|\cdot\|_{H}$ is denoted by $L_{H}^{2}(\mathbb{R})$. Note that this space contains the usual functions as well as distributions. Since $\mathcal{S}(\mathbb{R})$ is obviously a separable space with respect to the norm $\|\cdot\|_{H}$, we conclude that the space $L_{H}^{2}(\mathbb{R})$ possesses the same property. Further, let $\left\{e_{n}, n \geq 1\right\}$ be an orthonormal basis in the space $L_{H}^{2}(\mathbb{R})$.

The $n^{\text {th }}$ Hermite polynomial is defined by

$$
h_{n}(x):=(-1)^{n} \cdot e^{x^{2} / 2} \frac{d^{n}}{d x^{n}}\left(e^{-x^{2} / 2}\right) .
$$

Let the symbol $I$ stand for the set of finite multiindices, that is, for the set of sequences $\alpha=\left(\alpha_{1}, \alpha_{2}, \ldots\right)$ containing only a finite number of nonzero terms. Let

$$
\mathcal{K}_{\alpha}(\omega):=h_{\alpha_{1}}\left(\left\langle\omega, e_{1}\right\rangle\right) h_{\alpha_{2}}\left(\left\langle\omega, e_{2}\right\rangle\right) \ldots
$$

for $\alpha \in I$. 
Definition 2.1. The set of all $\psi(\omega)=\sum_{\alpha \in I} a_{\alpha} \mathcal{K}_{\alpha}(\omega) \in L^{2}\left(\mathrm{P}^{H}\right)$ such that the norm

$$
\|\psi\|_{H, k}^{2}:=\sum_{\alpha \in I} \alpha ! a_{\alpha}^{2}(2 \mathbb{N})^{k \alpha}
$$

is finite for all $k \in \mathbb{N}$ is called the Hida space of test functions $(\mathcal{S})_{H}$, where

$$
\gamma !=\prod_{j=1}^{\infty} \gamma_{j} !, \quad(2 \mathbb{N})^{\gamma}=\prod_{j=1}^{\infty}(2 j)^{\gamma_{j}}
$$

for $\gamma \in I$.

We introduce the Wick product in the space $(\mathcal{S})_{H}$ for two elements

$$
\phi(\omega)=\sum_{\alpha \in I} a_{\alpha} \mathcal{K}_{\alpha}(\omega) \text { and } \psi(\omega)=\sum_{\beta \in I} b_{\beta} \mathcal{K}_{\beta}(\omega)
$$

belonging to $(\mathcal{S})_{H}$ as follows:

$$
(\phi \diamond \psi)(\omega)=\sum_{\alpha, \beta \in I} a_{\alpha} b_{\beta} \mathcal{K}_{\alpha+\beta}(\omega)
$$

Now we define $\widehat{L}_{H}^{2}\left([0, T]^{n}\right)$ as the symmetric tensor product of $n$ copies of the space $L_{H}^{2}([0, T])$. By $\mathcal{L}_{n}$, we denote the set of all functions of $\widetilde{L}_{H}^{2}\left([0, T]^{n}\right.$ that are of the following form:

$$
f\left(s_{1}, \ldots, s_{n}\right)=\sum_{1 \leq k_{1}, \ldots, k_{n} \leq k} a_{k_{1}, \ldots, k_{n}} e_{k_{1}}\left(s_{1}\right) e_{k_{2}}\left(s_{2}\right) \cdots e_{k_{n}}\left(s_{n}\right) .
$$

For such an element, the $n$-tuple integral is defined by

$\mathcal{I}_{n}(f)=\sum_{1 \leq k_{1}, \ldots, k_{n} \leq k} a_{k_{1}, \ldots, k_{n}} \int_{0}^{T} e_{k_{1}}\left(s_{1}\right) d B_{s_{1}}^{H} \diamond \int_{0}^{T} e_{k_{2}}\left(s_{2}\right) d B_{s_{2}}^{H} \diamond \cdots \diamond \int_{0}^{T} e_{k_{n}}\left(s_{n}\right) d B_{s_{n}}^{H}$.

Each element of the space $\widehat{L}_{H}^{2}\left([0, T]^{n}\right)$ is the limit of a sequence of elements of $\mathcal{L}_{n}$. For an arbitrary element

$$
f \in \widehat{L}_{H}^{2}\left([0, T]^{n}\right),
$$

the integral $\mathcal{I}_{n}(f)$ is introduced as the limit of the integrals of a corresponding approximating sequence of functions belonging to $\mathcal{L}_{n}$ with the help of the following isometry:

$$
\mathrm{E}\left|I_{n}(f)\right|^{2}=n !\|f\|_{H}^{2} .
$$

Moreover, multiple integrals of different orders are orthogonal, that is,

$$
\mathrm{E}\left[\mathcal{I}_{n}(f) \mathcal{I}_{m}(g)\right]=n !\langle f, g\rangle_{H} \mathbb{I}(n=m) .
$$

This property allows one to expand random variables with respect to orthogonal multiple integrals.

Theorem 2.1 (Itô-Wiener expansion). Let $F \in L^{2}\left(\mathrm{P}^{H}\right)$. Then there are elements

$$
f_{n} \in \widehat{L}_{H}^{2}\left([0, T]^{n}\right), \quad n \geq 0,
$$

such that

$$
F(\omega)=\sum_{n=0}^{\infty} \mathcal{I}_{n}\left(f_{n}\right)
$$

where $\mathcal{I}_{0}\left(f_{0}\right):=\mathrm{E}[F]$. 


\section{MAin RESUlts}

Let $\xi \in L^{2}\left(\mathrm{P}^{H}\right)$. How precisely can $\xi$ be approximated by the increments of a fractional Brownian motion? Especially, what is the lower bound for the accuracy of such an approximation?

More precisely, given a partition $\pi=\left\{0=t_{0}<t_{1}<\cdots<t_{n}=T\right\}$, put

$$
\Delta_{\pi} B_{k}^{H}=B_{t_{k}}^{H}-B_{t_{k-1}}^{H} .
$$

We consider approximations of $\xi$ by the following functionals:

$$
F=F\left(\Delta_{\pi} B_{1}^{H}, \Delta_{\pi} B_{2}^{H}, \ldots, \Delta_{\pi} B_{n}^{H}\right) .
$$

Without loss of generality, one can assume that $\mathrm{E} \xi=0$ and $T=1$. To find a lower bound for the accuracy of approximation of $\xi$ by stochastic integrals one can restrict the consideration to the approximation of a stochastic integral of a nonrandom function by linear combinations of the increments

$$
\sum_{k=0}^{n-1} a_{k} \Delta_{\pi} B_{k}^{H}
$$

More specifically, the following result holds.

Lemma 3.1. Let $\xi \in L^{2}\left(\mathrm{P}^{H}\right)$ admit the Itô-Wiener expansion

$$
\xi=\sum_{n=1}^{\infty} \mathcal{I}_{n}\left(f_{n}\right)
$$

and let $G=G\left(\Delta_{\pi} B_{1}^{H}, \ldots, \Delta_{\pi} B_{n}^{H}\right)$ be an arbitrary functional of the increments. Then there are $a_{0}, a_{1}, \ldots, a_{n-1} \in \mathbb{R}$ such that

$$
\operatorname{Var}(\xi-G) \geq \operatorname{Var}\left(\mathcal{I}_{1}\left(f_{1}\right)-\sum_{k=0}^{n-1} a_{k} \Delta_{\pi} B_{k}^{H}\right) .
$$

Proof. Let

$$
G=\sum_{n=0}^{\infty} \mathcal{I}_{n}\left(g_{n}\right)
$$

be the Itô-Wiener expansion of $G$. The members of the Itô-Wiener expansion are orthogonal and, moreover,

$$
\begin{aligned}
\operatorname{Var}(\xi-G) & =\sum_{n \geq 1} n !\left\|f_{n}-g_{n}\right\|_{\widehat{L}_{H}^{2}\left([0, T]^{n}\right)}^{2} \geq\left\|f_{1}-g_{1}\right\|_{\widehat{L}_{H}^{2}([0, T])}^{2} \\
& =\operatorname{Var}\left(\mathcal{I}_{1}\left(f_{1}\right)-\mathcal{I}_{1}\left(g_{1}\right)\right) .
\end{aligned}
$$

Now we show that $\mathcal{I}_{1}\left(g_{1}\right)$ is a linear combination of increments. Note that $\mathcal{I}_{1}\left(g_{1}\right)$ is an orthogonal projection of $G$ onto the space $V_{1}$ of stochastic integrals of nonrandom functions. Let

$$
S=\sum_{k=0}^{n-1} a_{k} \Delta_{\pi} B_{k}^{H}
$$

be the orthogonal projection of $G$ onto the linear span $V$ of the increments $\Delta_{\pi} B_{k}^{H}$, $k=0, \ldots, n-1$, which is a subspace of $V_{1}$. By the three perpendiculars theorem, $S$ also is the orthogonal projection of $I\left(g_{1}\right)$ onto $V$. Thus the difference $I\left(g_{1}\right)-S$ is orthogonal to $V$, that is, it is orthogonal to all increments $\Delta_{\pi} B_{k}^{H}, k=0, \ldots, n-1$. Since these random variables are Gaussian, the difference $I\left(g_{1}\right)-S$ does not depend on the increments. In particular, $I\left(g_{1}\right)-S$ does not depend on $G$. This obviously implies that $I\left(g_{1}\right)-S=0$. 
In what follows we need the following property of the fractional Brownian motion that is called the property of asymptotically independent increments.

Theorem 3.1. Let $H>\frac{1}{2}$ and let $B^{H}=\left\{B^{H}, t \in[0, T]\right\}$ be the fractional Brownian motion with Hurst index $H$. Then

$$
\operatorname{Var}\left[\sum_{j=1}^{n} u_{j} \Delta_{\pi} B_{j}^{H}\right] \geq C \sum_{j=1}^{n} u_{j}^{2} \operatorname{Var}\left[\Delta_{\pi} B_{j}^{H}\right],
$$

where $u_{j} \in \mathbb{R}, j=1, \ldots, n$, are arbitrary real numbers and where $C$ is a certain constant that does not depend on $n$ and on $u_{j}, j=1, \ldots, n$.

Proof. First we recall that the increments of the fractional Brownian motion are stationary, and thus the covariance matrix of the increments of this stochastic process is a Töplitz matrix. Further, it is known that the spectral density of increments of the fractional Brownian motion is separated from zero in the case where the Hurst index is larger than $\frac{1}{2}$ (see [4]). Applying [5, Proposition 5.2.b)] we complete the proof of inequality (3).

First we obtain an upper bound for the distance between the integral $\int_{0}^{1} f(s) d B_{s}^{H}$ and the following smoothed version of an integral sum,

$$
S_{\pi_{\zeta}}(f):=\sum_{k=0}^{l-1} \frac{1}{\zeta} \int_{t_{k}}^{t_{k+1}} f(s) d s \cdot\left(B_{t_{k+1}}^{H}-B_{t_{k}}^{H}\right),
$$

that corresponds to the uniform partition $\pi_{\zeta}=\left\{0=t_{0}<t_{1}<\cdots<t_{l}=1\right\}$ of the diameter $\zeta=1 / l$.

Lemma 3.2. Let $H>\frac{1}{2}$ and let $f:[0,1] \rightarrow \mathbb{R}$ be a function for which the absolute values of the derivative are bounded from above by a number $M>0$. Then

$$
\mathrm{E}\left|\int_{0}^{1} f(s) d B_{s}^{H}-S_{\pi_{\zeta}}(f)\right|^{2} \leq M^{2} \zeta^{2} .
$$

Proof. In view of the isometry between $L^{2}\left(\mathrm{P}^{H}\right)$ for $L_{H}^{2}$ for integrals with respect to the fractional Brownian motion, the left hand side of inequality (41) equals

$$
\begin{aligned}
H(2 H-1) \cdot \frac{1}{\zeta^{2}} \sum_{k=0}^{l-1} \sum_{j=0}^{l-1} \int_{t_{k}}^{t_{k+1}} \int_{t_{j}}^{t_{j+1}} \int_{t_{k}}^{t_{k+1}} \int_{t_{j}}^{t_{j+1}} & |f(z)-f(t)| \\
& \times|f(u)-f(v)| \cdot|u-z|^{2 H-2} d v d t d u d z
\end{aligned}
$$

By the assumption, $f:|f(u)-f(v)| \leq M|u-v|$, and thus the latter double integral is bounded from above by

$$
\zeta^{2} \cdot M^{2} \cdot H(2 H-1) \int_{0}^{1} \int_{0}^{1}|s-t|^{2 H-2} d s d t=M^{2} \zeta^{2},
$$

which had to be proved.

In what follows we consider partitions $\pi_{\delta}=\left\{s_{k}^{\prime}=k / n\right\}_{k=0}^{n}$ of diameter $\delta=1 / n$ and sub-partitions $\pi_{\eta} \subseteq \pi_{\delta}, \pi_{\eta}=\left\{s_{i}=i / n^{m}\right\}_{i=0}^{n^{m}}$, of diameter $\eta=1 / n^{m}$, where $n$ is a sufficiently large number and $m$ is a certain positive integer number which will be specified later.

The following result provides a lower bound for the distance between the smoothed integral sums evaluated for a partition and for its sub-partition. 
Theorem 3.2. Let a function $f:[0,1] \rightarrow \mathbb{R}$ be such that $\left|f^{\prime}(x)\right| \leq M$ and

$$
\lambda\left\{x: f^{\prime}(x) \neq 0\right\}>0
$$

for all $x \in[0,1]$. Then

$$
\sum_{i=0}^{n-1} \sum_{k=i}^{i+n^{m-1}-1}\left(\frac{1}{\eta} \int_{s_{k}}^{s_{k+1}} f(s) d s-\frac{1}{\delta} \int_{s_{i}^{\prime}}^{s_{i+1}^{\prime}} f(t) d t\right)^{2} \geq K \delta^{2-m}
$$

where the constant $K$ does not depend on $n$ and on $m$.

Proof. Without loss of generality, we may assume that $\lambda\left\{x: f^{\prime}(x)>0\right\}>0$. Hence there exists a number $\varepsilon>0$ such that $\lambda\left\{x: f^{\prime}(x)>\varepsilon\right\}>0$. By the mean value theorem,

$$
\frac{1}{\eta} \int_{s_{k}}^{s_{k+1}} f(s) d s-\frac{1}{\delta} \int_{s_{i}^{\prime}}^{s_{i+1}^{\prime}} f(t) d t=\int_{\left[\theta^{i}, \theta_{k}^{i}\right]} f^{\prime}(x) d x,
$$

where $\theta^{i} \in\left[s_{i}^{\prime}, s_{i+1}^{\prime}\right]$ and $\theta_{k}^{i} \in\left[s_{k}, s_{k+1}\right]$. Further, we denote the left hand side of (5) by $S$ and put $A:=\left\{x: f^{\prime}(x)>\varepsilon\right\}$. For a given $\alpha \in(0,1 / 4)$, there exists an interval $[a, b] \subset[0,1]$ such that $\lambda(A \cap[a, b])>(b-a)(1-\alpha)$ by the Lebesgue density theorem. At least half of the intervals $\left[s_{i}^{\prime}, s_{i+1}^{\prime}\right]$ that belong to $[a, b]$ are such that

$$
\lambda\left(A \cap\left[s_{i}^{\prime}, s_{i+1}^{\prime}\right]\right)>\lambda\left(\left[s_{i}^{\prime}, s_{i+1}^{\prime}\right]\right)(1-2 \alpha) .
$$

Indeed, let $k_{1}$ be the total number of intervals for which inequality (6) holds, while $k_{2}$ is the total number of intervals for which inequality (6) fails. Then

$$
\lambda(A \cap[a, b]) \leq k_{1} \delta+k_{2} \delta(1-2 \alpha)
$$

or

$$
(b-a)(1-\alpha) \leq k_{1} \delta+\left(\frac{b-a}{\delta}-k_{1}\right) \delta(1-2 \alpha),
$$

which is equivalent to $(b-a) \alpha \leq 2 \alpha \delta k_{1}$. This implies that $k_{1} \geq(b-a) /(2 \delta)$ and thus completes the proof.

Further,

$$
\begin{aligned}
S & \geq \sum^{\prime} \sum_{k=i}^{i+n^{m-1}-1}\left(\int_{\left[\theta^{i}, \theta_{k}^{i}\right]} f^{\prime}(s) d s\right)^{2} \\
& \geq \sum^{\prime} \sum_{k=i}^{i+n^{m-1}} \frac{1}{2}\left(\int_{\left[\theta^{i}, \theta_{k}^{i}\right] \cap A} f^{\prime}(x) d x\right)^{2}-\left(\int_{\left[\theta^{i}, \theta_{k}^{i}\right] \cap A^{c}} f^{\prime}(x) d x\right)^{2},
\end{aligned}
$$

where the symbol $\sum^{\prime}$ means the summation for those $i=0, \ldots, n-1$ for which (6) holds. Now we estimate

$$
\sum_{k=i}^{i+n^{m-1}}\left(\int_{\left[\theta^{i}, \theta_{k}^{i}\right] \cap A} f^{\prime}(x) d x\right)^{2}
$$

and

$$
\sum_{k=i}^{i+n^{m-1}}\left(\int_{\left[\theta^{i}, \theta_{k}^{i}\right] \cap A^{c}} f^{\prime}(x) d x\right)^{2}
$$

separately. 
For the first sum, we denote by $\kappa:=\delta / \eta$ the total number of the intervals of the partition $\pi_{\eta}$ belonging to each interval of the partition $\pi_{\delta}$. Then

$$
\begin{aligned}
\sum_{k=i}^{i+n^{m-1}}\left(\int_{\left[\theta^{i}, \theta_{k}^{i}\right] \cap A} f^{\prime}(x) d x\right)^{2} & \geq \sum_{k=i}^{i+\kappa-1} \varepsilon^{2} \lambda^{2}\left(\left[\theta^{i}, \theta_{k}^{i}\right] \cap A\right) \\
& \geq \varepsilon^{2} \frac{1}{\kappa}\left(\sum_{k=i}^{i+\kappa} \lambda\left(\left[\theta^{i}, \theta_{k}^{i}\right] \cap A\right)\right)^{2} .
\end{aligned}
$$

Let $\theta^{i} \in\left[s_{m}, s_{m+1}\right]$. Then the latter expression is not less than

$$
\begin{aligned}
& \frac{1}{\kappa}\left(\sum_{k=i}^{i+m-1} \lambda\left(\left[s_{k+1}, s_{m}\right] \cap A\right)+\sum_{k=i+m}^{\kappa} \lambda\left(\left[s_{m+1}, s_{k}\right] \cap A\right)\right)^{2} \\
& \quad=\frac{1}{\kappa}\left(\sum_{k=1}^{m-1} k \lambda\left(\left[s_{k}, s_{k+1}\right] \cap A\right)+\sum_{k=m}^{\kappa-2}(\kappa-k+1) \lambda\left(\left[s_{k}, s_{k+1}\right] \cap A\right)\right)^{2} \\
& \quad \geq C_{1} \frac{1}{\kappa}(1-4 \alpha)^{2} \eta^{2} \kappa^{4}
\end{aligned}
$$

The latter inequality holds, since

$$
\lambda\left(\left[s_{k}, s_{k+1}\right] \cap A\right) \geq \lambda\left(\left[s_{k}, s_{k+1}\right]\right)(1-4 \alpha)
$$

for at least half of the intervals $\left[s_{k}, s_{k+1}\right]$ with $k=i, \ldots, i+\kappa-1$.

In view of inequality (6),

$$
\sum_{k=i}^{i+\kappa-1}\left(\int_{\left[\theta^{i}, \theta_{k}^{i}\right] \cap A^{c}} f^{\prime}(x) d x\right)^{2} \leq M^{2} 4 \alpha^{2} \delta^{2} \cdot \delta \eta^{-1} .
$$

Here we have used the property that $f^{\prime}(x) \leq\left|f^{\prime}(x)\right| \leq M$ and that the integral of a positive function over the interval $\left[\theta^{i}, \theta_{k}^{i}\right]$ does not exceed the corresponding integral over the interval $\left[s_{i}^{\prime}, s_{i+1}^{\prime}\right]$. It is clear that

$$
C_{1}(1-4 \alpha)^{2}-M^{2} 4 \alpha^{2}>C_{2}>0
$$

for an appropriate $\alpha$, whence

$$
S>\frac{b-a}{2 \delta} \delta^{3} \eta^{-1} C_{2}=C_{3} \delta^{2} \eta^{-1}=C_{3} \delta^{2-m} .
$$

The proof is complete.

Now we are in a position to prove the main result.

Theorem 3.3. Let a random variable $\xi$ be such that $\xi \in L^{2}\left(\mathrm{P}^{H}\right)$. If the function $f_{1}$ in its Itô-Wiener expansion (2) is differentiable and such that

$$
\begin{gathered}
\left|f^{\prime}(x)\right| \leq M, \quad x \in[0,1], \\
\lambda\left\{x \in[0,1]: f^{\prime}(x) \neq 0\right\}>0,
\end{gathered}
$$

then there exists a constant $C>0$ such that

$$
\mathrm{E}\left[(\xi-F)^{2}\right] \geq C \delta^{4 H}
$$

for an arbitrary uniform partition $\pi=\pi_{\delta}=\left\{0=t_{0}<t_{1}<\cdots<t_{n}=1\right\}$ of a sufficiently small diameter $\delta$ and for all functionals $F$ of increments of the fractional Brownian motion defined on this partition. 
Proof. The Itô-Wiener expansion of the random variable $\xi$ is given by

$$
\xi=\sum_{n=0}^{\infty} \mathcal{I}_{n}\left(f_{n}\right)
$$

By Lemma 3.1, there exists a sequence of numbers $a_{0}, a_{1}, \ldots, a_{n-1} \in \mathbb{R}$ such that

$$
\operatorname{Var}(\xi-F) \geq \operatorname{Var}\left(\mathcal{I}_{1}\left(f_{1}\right)-\sum_{k=0}^{n-1} a_{k} \Delta_{\pi} B_{k}^{H}\right) .
$$

To simplify the notation, put $f=f_{1}$. In fact, we need to estimate the deviation between

$$
\mathcal{I}_{1}(f)=\int_{0}^{1} f(s) d B_{s}^{H}
$$

and

$$
\sum_{k=0}^{n-1} a_{k}\left(B_{s_{k+1}^{\prime}}^{H}-B_{s_{k}^{\prime}}^{H}\right) .
$$

We split each interval of the partition $\pi_{\delta}$ into $\delta / \eta$ intervals of length $\eta$. Further,

$$
\begin{aligned}
& \mathrm{E}\left[\left(\int_{0}^{1} f(s) d B_{s}^{H}-\sum_{k=0}^{n-1} a_{k}\left(B_{s_{k+1}^{\prime}}^{H}-B_{s_{k}^{\prime}}^{H}\right)\right)^{2}\right] \\
& \quad=\mathrm{E}\left[\left(\int_{0}^{1} f(s) d B_{s}^{H}-S_{\pi_{\eta}}(f)+S_{\pi_{\eta}}(f)-\sum_{k=0}^{n-1} a_{k}\left(B_{s_{k+1}^{\prime}}^{H}-B_{s_{k}^{\prime}}^{H}\right)\right)^{2}\right] \\
& \quad \geq \frac{1}{2} \mathrm{E}\left[\left(S_{\pi_{\eta}}(f)-\sum_{k=0}^{n-1} a_{k}\left(B_{s_{k+1}^{\prime}}^{H}-B_{s_{k}^{\prime}}^{H}\right)\right)^{2}\right]-\mathrm{E}\left[\left(\int_{0}^{1} f(s) d B_{s}^{H}-S_{\pi_{\eta}}(f)\right)^{2}\right] \\
& =: D_{1}+D_{2},
\end{aligned}
$$

where

$$
S_{\pi_{\eta}}(f)=\sum_{k=0}^{n^{m}-1} \frac{1}{\eta} \int_{s_{k}}^{s_{k+1}} f(u) d u\left(B_{s_{k+1}}^{H}-B_{s_{k}}^{H}\right) .
$$

First we estimate from below the term $D_{1}$ that can be rewritten as follows:

$$
D_{1}=\mathrm{E}\left|\sum_{k=0}^{n^{m}-1} \frac{1}{\eta} \int_{s_{k}}^{s_{k+1}} f(s) d s \cdot \triangle B_{s_{k}}^{H}-\sum_{k=0}^{n-1} a_{k} \triangle B_{s_{k}^{\prime}}^{H}\right|^{2} .
$$

Note that each interval

$$
\left[s_{k}^{\prime}, s_{k+1}^{\prime}\right), \quad k=0, \ldots, n-1,
$$

contains $n^{m-1}$ points $s_{i}, i=k, \ldots, k+n^{m-1}-1$. Hence

$$
\Delta=\mathrm{E}\left|\sum_{k=0}^{n^{m}-1}\left(\frac{1}{\eta} \int_{s_{k}}^{s_{k+1}} f(s) d s-a_{\left[\frac{k}{n^{m}-1}\right]}\right) \Delta B_{s_{k}}^{H}\right|^{2} .
$$

Applying Theorem 3.1 we get

$$
\begin{aligned}
\Delta & \geq C \cdot \eta^{2 H} \cdot \sum_{k=0}^{n^{m}-1}\left(\frac{1}{\eta} \int_{s_{k}}^{s_{k+1}} f(s) d s-a_{\left[\frac{k}{n m-1}\right]}\right)^{2} \\
& =C \delta^{2 m H} \sum_{k=0}^{n-1} \sum_{i=k}^{k+n^{m-1}-1}\left(\frac{1}{\eta} \int_{s_{i}}^{s_{i+1}} f(s) d s-a_{k}\right)^{2} .
\end{aligned}
$$


Minimizing each sum

$$
\sum_{i=k}^{k+n^{m-1}-1}\left(\frac{1}{\eta} \int_{s_{i}}^{s_{i+1}} f(s) d s-a_{k}\right)^{2}
$$

with respect to $a_{k}, k=0, \ldots, n-1$, we prove that the latter double sum is not less than its value calculated for the sequence

$$
a_{i}=\frac{1}{n^{m-1}} \cdot \frac{1}{\eta} \sum_{k=i}^{i+n^{m-1}-1} \int_{s_{k}}^{s_{k+1}} f(u) d u=\frac{1}{\delta} \int_{s_{i}^{\prime}}^{s_{i+1}^{\prime}} f(u) d u
$$

namely

$$
D_{1} \geq C \delta^{2 m H} \sum_{k=0}^{n-1} \sum_{i=k}^{k+n^{m-1}-1}\left(\frac{1}{\eta} \int_{s_{k}}^{s_{k+1}} f(s) d s-\frac{1}{\delta} \int_{s_{k}^{\prime}}^{s_{k+1}^{\prime}} f(t) d t\right)^{2} .
$$

By Theorem 3.2. $D_{1} \geq C_{1} \delta^{2 m H-m+2}$. On the other hand, $D_{2} \leq-C_{2} \delta^{2 m}$ by Lemma 3.2 applied to the partition $\pi_{\eta}$. Thus

$$
\mathrm{E}\left[\left(\int_{0}^{1} f(s) d B_{s}^{H}-\sum_{k=0}^{n-1} a_{k}\left(B_{s_{k+1}^{\prime}}^{H}-B_{s_{k}^{\prime}}^{H}\right)\right)^{2}\right] \geq C_{1} \delta^{2 m H-m+2}-C_{2} \delta^{2 m} .
$$

Putting $m=2$ (this gives the best estimate), we prove that the latter expression is not less than $C_{1} \delta^{4 H}$ for sufficiently small $\delta$. Theorem 3.3 is proved.

Remark 3.1. If $H=\frac{1}{2}$, the accuracy of approximation in the Milstein scheme is of order $\delta^{2}=\delta^{4 H}$; that is, the estimate of the order of accuracy obtained in Theorem 3.3 is precise if $H=\frac{1}{2}$ (note however that Theorem 3.3 is proved for $H>\frac{1}{2}$ only). The question on whether the estimate in Theorem 3.3 is precise for $H>\frac{1}{2}$ is still under consideration.

Example 3.1. Consider the Ornstein-Uhlenbeck equation with the fractional Brownian motion

$$
X_{t}=X_{0}+\int_{0}^{t}\left(a X_{s} d s+b d B_{s}^{H}\right), \quad t \in[0,1],
$$

where $X_{0}, a \in \mathbb{R}$, and $b>0$.

It is well known that

$$
X_{t}=X_{0} e^{a t}+b \int_{0}^{t} e^{a(t-s)} d B_{s}^{H}, \quad t \in[0,1]
$$

is a solution of this equation.

Let $\xi=X_{1}$. Then equality (8) provides the Itô-Wiener expansion for $\xi$, namely

$$
f_{0}=X_{0} e^{a}, \quad f_{1}(t)=b e^{a(1-t)}, \quad f_{n}=0, \quad n \geq 2 .
$$

It is easy to see that the derivative of the function $f_{1}$ is bounded and does not vanish. Hence all the assumptions of Theorem 3.3 hold for $\xi$.

\section{Concluding Remarks}

The problem on the approximation of random variables by functionals of increments of the fractional Brownian motion is studied in this paper. A lower bound for the accuracy of the approximation is obtained for a certain class of random variables (namely, for random variables whose Itô-Wiener expansion is such that the function $f_{1}$ is not constant). 


\section{BIBLIOGRAPHY}

1. F. Biagini, Y. Hu, B. Oksendal, and T. Zhang, Stochastic Calculus for Fractional Brownian Motion and Applications, Springer, Berlin, 2008. MR 2387368 (2010a:60145)

2. J. M. C. Clark and R. J. Cameron, The maximum rate of convergence of discrete approximations for stochastic differential equations, Stochastic Differential Systems Filtering and Control, Lecture Notes in Control and Information Sciences, vol. 25, 1980, no. 1, pp. 162-171. MR609181 (82f:60133)

3. A. Deya, A. Neuenkirch, and S. Tindel, A Milstein-type scheme without Lévy area terms for SDEs driven by fractional Brownian motion, Ann. Inst. Henri Poincaré, Probab. Stat. 48 (2012), no. 2, 518-550. MR2954265

4. T. Dieker and M. Mandjes, On spectral simulation of fractional Brownian motion, Probab. Engrg. Inform. Sci. 17 (2003), no. 3, 417-434. MR1984656 (2004c:60231)

5. U. Grenander and G. Szegö, Toeplitz Forms and their Applications, Chelsea Publishing Company, New York, 1984. MR890515 (88b:42031)

6. Yu. Mishura and G. Shevchenko, The rate of convergence for Euler approximations of solutions of stochastic differential equations driven by fractional Brownian motion, Stochastics 80 (2008), no. 5, 489-511. MR2456334(2009k:60138)

7. I. Nourdin and A. Neuenkirch, Exact rate of convergence of some approximation schemes associated to SDEs driven by a fractional Brownian motion, J. Theoret. Probab. 20 (2007), no. 4, 871-899. MR2359060 (2009e:60150)

8. A. Neuenkirch, Optimal pointwise approximation of stochastic differential equations driven by fractional Brownian motion, Stoch. Process. Appl. 118 (2008), no. 12, 2294-2333. MR2474352 (2010d:60152)

Department of Probability Theory, Statistics, and Actuarial Mathematics, Faculty for Mechanics and Mathematics, National Taras Shevchenko University, Academician Glushkov Avenue, 4E, Kiev 03127, Ukraine

E-mail address: zhora@univ.kiev.ua

Department of Probability Theory, Statistics, and Actuarial Mathematics, Faculty for Mechanics and Mathematics, National Taras Shevchenko University, Academician Glushkov Avenue, 4E, Kiev 03127, Ukraine

E-mail address: tarasenya@gmail.com

Received 23/NOV/2011

Translated by N. SEMENOV 